\title{
Pediatric intracranial hypertension
}

\author{
Experience from 2 Tertiary Centers
}

Fahad A. Bashiri, MD, Hissah K. Al Abdulsalam, MD, Saeed M. Hassan, MD, Waleed A. Al Twaijri, MD, Fuad I. Almalki, MD, Amal Y. Kentab, MD, Muddathir H. Hamad, MD, Ali H. Alwadei, MD, FRCPC, Daniah A. Al-Showaeir, MD, Ikhlass S. Altweijri, MD, Haifa M. Aldabjan, MD, Moudi S. Aldegether, MD, Abdulrahman A. Albakr, MD, Wajda M. Alhothali, MD, Abdulrazag M. Ajlan, MD, FRCSC, Hamdy H. Hassan, MD, Mustafa A. Salih, FRCPCH, FAAN.

\begin{abstract}
الأهداف : تهدف هذه الدراسة إلى مراجعة تجربة إثنين من مراكز

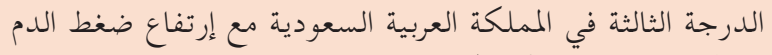
داخل القحف عند الأطفال.

المنهجية : قمنا بمراجعة وتحليل المرضي الذين تم تشخيصهم بإِرتفاع

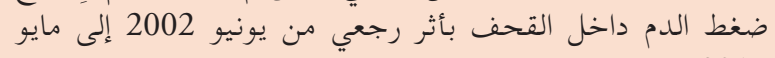
2017

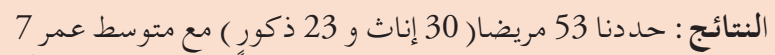

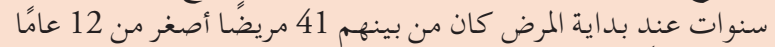

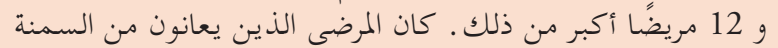

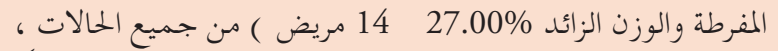
8 منهم (66.7\%) أكبر من 12 سنة . كانت الأعراض الأكثر شيوعًا

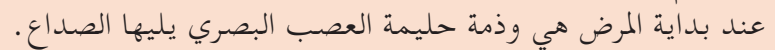

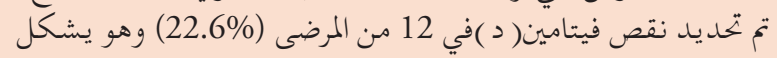

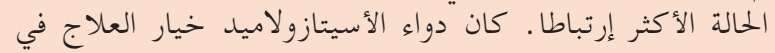

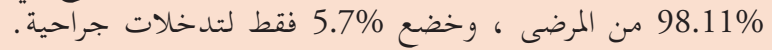
تراوحت مدة المتابعة من 6 أشهر إلى 8 سنى سنوات .

الحلاصة : إرتفاع ضغط الدم داخل الجمجمة نادر عندالأطفال

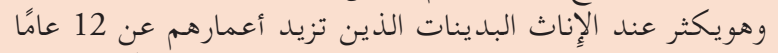

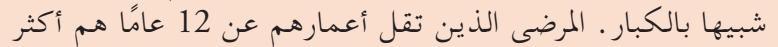

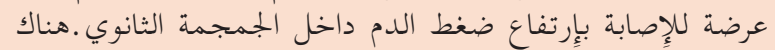

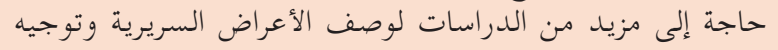
خطة المعالجة . حالى مزئ.
\end{abstract}

Objectives: To review the experience of 2 tertiary centers in Saudi Arabia with intracranial hypertension (IH) in the pediatric population.

Methods: We retrospectively reviewed and analyzed pediatric patients diagnosed with IH from June 2002 to May 2017 in 2 institutes.
Results: We identified 53 patients (30 females and 23 males) with a mean age of 7 years at the time of presentation. Among them, 41 patients were younger than 12 years, and 12 were older. Obese and overweight patients constituted $27.00 \%(n=14)$ of all cases, $8(66.7 \%)$ of whom were older than 12 years. The most common presenting feature was papilledema followed by headache. Vitamin D deficiency, which constituted the most common associated condition, was identified in $12(22.6 \%)$ patients. Acetazolamide was the treatment option in $98.11 \%$ of patients, and only $5.7 \%$ underwent surgical interventions. The length of follow-up ranged from 6 months to 8 years.

Conclusion: Intracranial hypertension is rare in children and commonly seen in overweight females older than 12 years similar to adults. Patients younger than 12 years tend to develop secondary IH. More studies are needed to characterize the clinical presentation and guide the management plan.

Neurosciences 2019; Vol. 24 (4): 257-263 doi: 10.17712/nsj.2019.4.20190052

From the Division of Neurology (Bashiri, Hassan, Kentab, Hamad, Salih), Departments of Pediatrics, Department of Surgery (Al Abdulsalam, Altweijri, Aldabjan, Aldegether, Albakr, Alhothali, Ajlan), Neuro-ophthalmology Division (Al-Showaeir), Department of Ophthalmology, and from the Neuroradiology Division (Hassan), Department of Radiology, College of Medicine, King Saud University, and from Department of Pediatric Neurology (Alwadei), National Neuroscience Institute, King Fahad Medical City, and from King Abdullah Children Specialist Hospital (Al Twaijri), National Guard Health Affairs, Riyadh, and from the Department of Pediatrics (Almalki), College of Medicine, Majmaah University, Almajmaah, Kingdom of Saudi Arabia.

Received 12th June 2019. Accepted 13th August 2019.

Address correspondence and reprint request to: Dr. Fahad A. Bashiri, Division of Pediatric Neurology, Department of Pediatrics, College of Medicine, King Saud University, Riyadh, Kingdom of Saudi Arabia. E-mail:fbashiri@ksu.edu.sa

ORCID ID: https://orcid.org/0000-0003-1436-6727 
$\mathrm{I}$ ntracranial hypertension (IH) is rarely reported in children. It is characterized by increased intracranial pressure (ICP) without any evidence of underlying brain pathology, structural abnormalities, hydrocephalus, or any abnormal meningeal enhancement. ${ }^{1}$ The incidence of $\mathrm{IH}$ differs from region to region due to variations in the prevalence of obesity and other secondary causes. The annual incidence of $\mathrm{IH}$ in children is 0.9 per 100,000 in the United States, ${ }^{2} 0.5$ per 100,000 in Germany, ${ }^{3} 0.6$ per 100,000 in Nova Scotia and Prince Edward Island in Eastern Canada, ${ }^{4}$ and 1.2 per 100,000 in Croatia. ${ }^{5}$ A study carried out in Oman estimated the incidence of $\mathrm{IH}$ to be 1.9 per 100,000 in children below 15 years of age; with it being higher in female children. ${ }^{6}$ The present study aimed to review the clinical presentation, possible aetiological factors, diagnosis, management, and outcomes in children with $\mathrm{IH}$ in 2 tertiary institutes in Saudi Arabia.

Methods. After approval of the study proposal by the Research Ethics Committee at King Saud University, and King Abdullah Research Center, we did a retrospective review of all pediatric patients diagnosed with IH between June 2002 to May 2017 at King Saud University Medical City and King Abdullah Children Specialist Hospital, National Guard Health Affairs. Patients with identified aetiologies were classified as having secondary $\mathrm{IH}(\mathrm{SIH})$, and patients without identified aetiologies were classified as having idiopathic or primary IH (PIH). The following parameters were recorded: patients' characteristics, age at the time of presentation, presenting symptoms and signs, associated conditions, diagnostic procedures, brain images, treatment, and outcome. IH was defined based on the modified Dandy criteria. ${ }^{7}$ The inclusion criteria were: patients aged between 6 months and 18 years, normal brain magnetic resonance imaging (MRI) and/ or computed tomographic (CT) scan, a cerebrospinal fluid (CSF) opening pressure greater than $250 \mathrm{~mm}$ $\mathrm{H}_{2} \mathrm{O}$ with normal CSF analysis, bilateral papilledema, and intact neurologic examination except for isolated abducent, trochlear or oculomotor paresis. We excluded patients who had abnormal CSF study results, normal or undocumented ICP pressure, brain imaging showing structural abnormalities, and abnormal neurological examination other than sixth nerve palsy or papilledema.

Disclosure. Authors have no conflict of interests, and the work was not supported or funded by any drug company.
We identified a total of 64 cases; 11 were excluded for missing data, lack of documentation, or inability to meet the inclusion criteria, thus resulting in the inclusion of a final 53 patients. We classified the patients as either prepubertal or pubertal. The use of secondary sexual characteristics is often the proper method to classify children as either pubertal or prepubertal. However, due to the retrospective nature of this study, we instead chose an average age. Therefore, patients younger than 12 years were considered prepubertal, while patients older than 12 years were considered pubertal. Based on their body mass index (BMI), we further classified the patients into three groups: obese, overweight, or normal body weight. A child was described as obese if the BMI was more than 30 and overweight if the BMI ranged between 25 and 29.9.

Statistical analysis was performed using IBM SPSS Statistics for Windows, version 19 (IBM Corp., Armonk, N.Y., USA).

Results. A total of 53 children with $\mathrm{IH}$ were analyzed in this study. The mean age of the patients at presentation was 7.7 years, ranging from 8 months to 16 years. Twenty-three children were male and thirty were female. Most individuals $(\mathrm{n}=36$ [69.2\%]) had normal weight, $7(13.5 \%)$ were overweight, and 7 (13.5\%) were obese. The demographics of the participants are shown in Table 1. In the present study, 12 patients were 12 years and older (pubertal group) and 41 patients were younger than 12 years (prepubertal group). The mean age at presentation in the pubertal group was $13.66 \pm 1.66$ years (range $=12-16$ years) and $5.9 \pm 3.38$ years (range $=8$ months -11 years) in the prepubertal group. The percentage of females in the prepubertal group was $52.63 \%$ and pubertal group was $66.66 \%$. Obese and overweight patients constituted $27.00 \%$ $(n=14)$ of all cases, and $66.7 \%(n=8)$ of those in the

Table 1 - Demographics of the participants.

\begin{tabular}{lc}
\hline Demographics & $\mathbf{n}(\%)$ \\
\hline Age (prepubertal group) & \\
<1 year & $3(7.31)$ \\
1-6 years & $20(48.78)$ \\
>6years & $18(43.9)$ \\
Sex (total group) & \\
Male & $23(43.4)$ \\
Female & $30(56.6)$ \\
BMI (total group) & \\
Normal & $36(69.2)$ \\
Overweight & $7(13.5)$ \\
Obese & $7(13.5)$ \\
Asymptomatic Papilledema & $16(30.2)$ \\
Symptomatic Papilledema & $35(66.0)$ \\
\hline
\end{tabular}


Table 2 - Associated conditions and medications (Secondary Intracranial Hypertension).

\begin{tabular}{lc}
\hline Associated conditions & $\mathbf{n}(\%)$ \\
\hline Vitamin D deficiency & $12(22.6)$ \\
Craniosynostosis & $4(7.5)$ \\
Down syndrome & $4(7.5)$ \\
ADHD & $2(3.8)$ \\
Spina bifida & $2(3.8)$ \\
Achondroplasia & $1(1.9)$ \\
Acute lymphoblastic leukaemia & $1(1.9)$ \\
Atrioseptal Defect & $1(1.9)$ \\
Autoimmune thyroiditis & $1(1.9)$ \\
Bronchial asthma & $1(1.9)$ \\
Cataract & $1(1.9)$ \\
Mucopolysaccharidosis type VI & $1(1.9)$ \\
Middle East Syndrome & $1(1.9)$ \\
Minimal change disease & $1(1.9)$ \\
MLC 1 gene mutation & $1(1.9)$ \\
Obstructive sleep apnoea & $1(1.9)$ \\
Epilepsy during infancy & $1(1.9)$ \\
Retinitis pigmentosa & $1(1.9)$ \\
Skeletal dysplasia & $1(1.9)$ \\
Subaortic stenosis & $1(1.9)$ \\
Sagittal “Dural” sinus thrombosis & $1(1.9)$ \\
Medications & \\
Carbamazepine & $2(3.77)$ \\
Growth hormone & $1(1.9)$ \\
Steroid & $1(1.9)$ \\
Cyclosporin & $1(1.9)$ \\
Methotrexate & $1(1.9)$ \\
\hline ADHD - Attention deficit hyperactivity disorder \\
\hline
\end{tabular}

Table 3 - Surgical treatment and recurrence.

\begin{tabular}{lc}
\hline Surgical treatment \& recurrence & $\mathbf{n}(\%)$ \\
\hline Surgical treatment & \\
Yes & $3(5.7)$ \\
No & $50(94.3)$ \\
Recurrence & \\
Yes & $7(13.2)$ \\
No & $46(86.8)$ \\
\hline
\end{tabular}

pubertal group (Table 1). Compared to those in the pubertal group, most patients in the prepubertal group $(n=34[85 \%])$ were underweight or had normal weight. The prepubertal group was further categorized into three groups based on age, those less than one year old at diagnosis $(n=3[7.3 \%])$, those between 1 and 6 years old $(n=20[48.8 \%])$, and those older than 6 years $(\mathrm{n}=18[43.9 \%])$.

The most common clinical feature was papilledema $(\mathrm{n}=52[98.1 \%])$ followed by headache $(\mathrm{n}=42[79.2 \%])$.
Papilledema was asymptomatic in $16(30.2 \%)$ patients. Other common symptoms included visual problems such as photophobia, diplopia, and blurred vision. Neurologic examination was reported as normal in 29 (54.71\%) patients, and sixth nerve palsy was present in $4(7.54 \%)$ patients. All the patients had an insignificant perinatal history, except 2 who were born prematurely at 30 and 34 weeks of gestation. The clinical features of the patients are shown in Table 1 . Figure $1 \& 2$ demonstrates papilledema in some of the patients.

Out of the 53 patients, 13 patients had PIH (idiopathic) and 40 patients had SIH (Table 2). There were no significant differences in gender, age at presentation, or BMI between the PIH and SIH patients. Interestingly, $16(44.4 \%)$ patients with $\mathrm{SIH}$ required additional lumbar puncture (LP). In terms of outcome, the recurrence in the SIH group was high ( $\mathrm{n}=6[19.4 \%]$ ), whereas only one patient had a recurrence in the PIH group. At the time of presentation, majority of the patients $(n=47[88.7 \%])$ were not on any medications except for $2(3.77 \%)$ who were on carbamazepine, one $(1.9 \%)$ who received growth hormone therapy, one $(1.9 \%)$ on steroids and cyclosporine, and one (1.9\%) on methotrexate (Table 2). Lumbar puncture was carried out in all cases. Opening pressure was high in all patients. Cerebrospinal fluid analysis was normal in all patients. All patients underwent neuroimaging studies, including CT and/or MRI brain scans. Four (7.5\%) patients had craniosynostosis. Magnetic resonance venography (MRV) had been performed in 37 (69.8\%) patients. Sagittal dural sinus thrombosis was evident in one patient. The MRI showed typical IH features in more than half of the patients $36(67.9 \%)$. Figure 3 demonstrates the findings in one of the patients. Further laboratory tests were performed in most patients and included the thyroid function test, antinuclear antibody test, and C3, C4 and $25(\mathrm{OH})$ vitamin D levels. All these tests showed normal results except for $25(\mathrm{OH})$ vitamin $\mathrm{D}$ levels, which were low in $12(22.6 \%)$ patients.

The majority of patients $(\mathrm{n}=52[98.1 \%])$ were treated with acetazolamide as first-line therapy. Only one patient was treated with topiramate alone. Out of the 52 patients who received acetazolamide, 29 (54.71\%) did not require further treatment. Most patients $(\mathrm{n}=50$ [94.3\%]) did not require surgical intervention, and only $3(5.7 \%)$ patients underwent surgical treatment (lumboperitoneal shunt). According to the long term outcome, there were no relapses in $46(86.8 \%)$ patients. None of our patients had optic nerve fenestration. The surgical treatment and recurrence are summarized in Table 3. The length of follow-up ranged from 6 months to 8 years. 

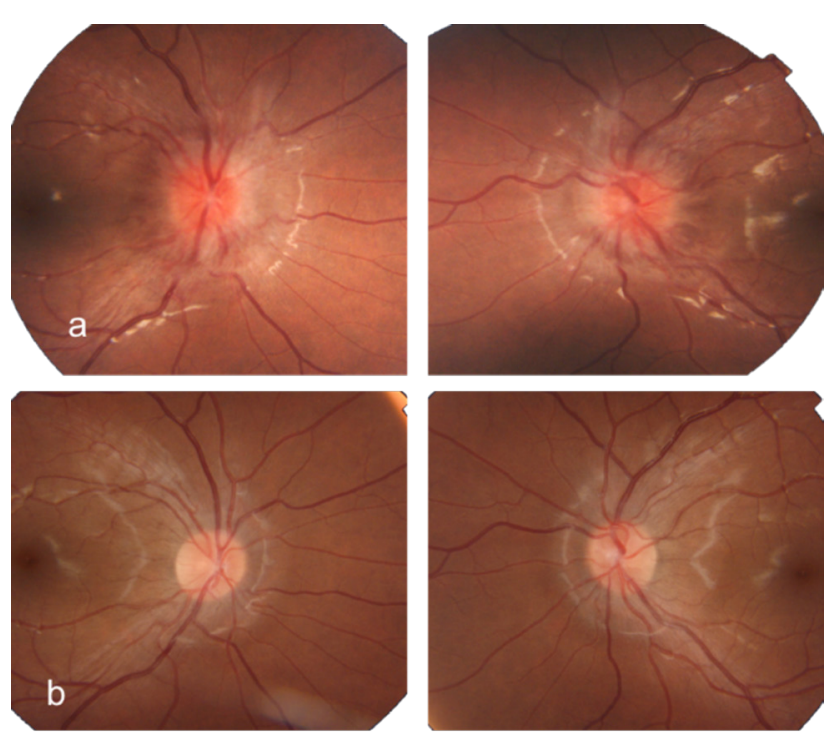

Figure 1 - Fundus photographs of a 9-year-old girl showing her optic nerves. A) At presentation, the right and left optic nerves show papilledema. B) The right and left optic discs with resolving papilledema a few months later after medical treatment.

Discussion. The $\mathrm{IH}$ is typically present in overweight women; however, it can occur at any age. Unlike in adults, several studies have found that IH in prepubertal children is less commonly associated with obesity, and has no female predominance. ${ }^{8,9}$ On the other hand, IH in postpubertal children is usually similar to that in adults, and is associated with overweight and commonly occurs in women. ${ }^{10}$ In our study, obese and overweight patients constituted $27.00 \%(n=14)$ of all cases, a majority of whom were pubertal. This classification is rarely done in pediatric IH cases reported in the literature.

As the name implies, the pathophysiology of IH is not fully understood. There are several proposed hypotheses such as increased CSF production, decreased CSF outflow, increase in cerebral blood volume, increase in water content, venous obstruction, chronic inflammation, and metabolic causes. ${ }^{9}$ Obesity is an important risk factor for $\mathrm{IH}$ in postpubertal females. ${ }^{11}$ However, the absence of obesity and sex predilection in some prepubertal children and adolescents with IH suggests a different pathogenesis in these patients. Hence, a secondary cause should be carefully investigated and ruled out before making a diagnosis. ${ }^{4,11,12}$

Additionally, IH can also be secondary to an underlying medical condition or use of certain medications. In a recent study of $\mathrm{IH}$ in the pediatric population, $30 \%$ of the cases were found to have
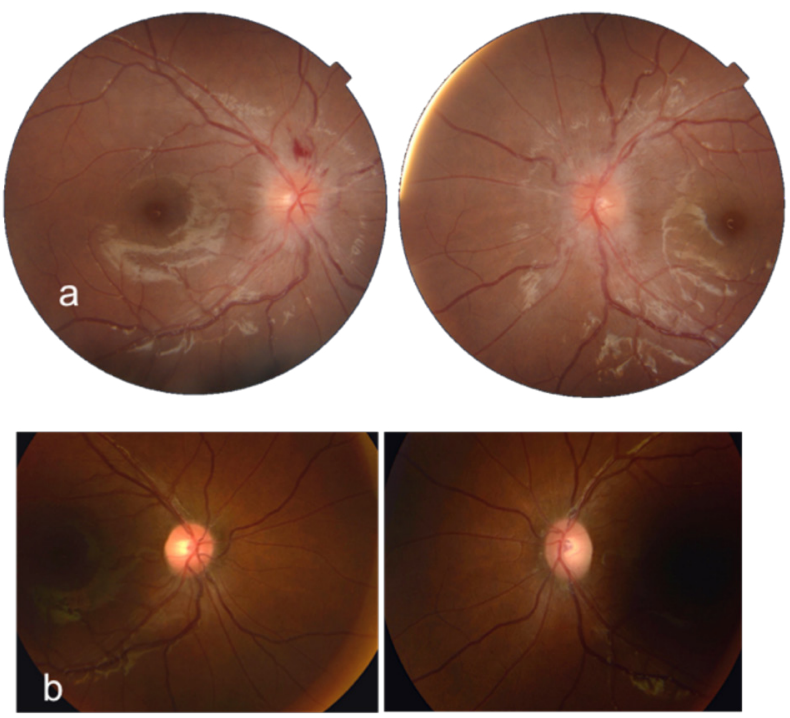

Figure 2 - Fundus photographs of a 12-year-old girl showing her optic nerves. A) At presentation, the right and left optic nerves show papilledema with peripapillary flame-shaped hemorrhages seen in the right eye indicating an acute process. B) Flat right and left optic discs a few months after medical treatment.

a secondary cause. ${ }^{11}$ Several factors have been associated with $\mathrm{IH}$ in pediatric patients. These include hyperparathyroidism, thyroid replacement therapy, treatment with recombinant human growth hormone, Chiari malformation, meningitis, hydrocephalus, craniosynostosis, traumatic brain injury, superior sagittal sinus thrombosis, leukemia, congestive heart failure, renal failure, and kidney transplantation. ${ }^{11,12}$ In a recent study, vitamin $\mathrm{D}$ deficiency was found in $26.3 \%$ of pediatric IH patients, with none of them having hypocalcemia or signs and symptoms of rickets. ${ }^{13}$ In our study, $12(22.6 \%)$ cases were found to be associated with vitamin $\mathrm{D}$ deficiency. The exact pathophysiology of increased intracranial pressure in relation to vitamin $\mathrm{D}$ deficiency is not fully known. In the present study, one case of hypoparathyroidismretardation-dysmorphism syndrome, also known as Middle east syndrome or Sanjad-Sakati syndrome (OMIM \# 241410), had IH possibly resulting from the associated hypoparathyroidism, a recognized risk factor of $\mathrm{IH}^{14}$

The most common clinical presentation in children is headache. ${ }^{11,15,16}$ Other clinical features include nausea and vomiting, blurred vision, and double vision. The neurological examination of children with IH is usually normal except for papilledema, decreased visual acuity, visual fields defects, neck stiffness, and sixth nerve palsy. Although papilledema is an important sign in children 

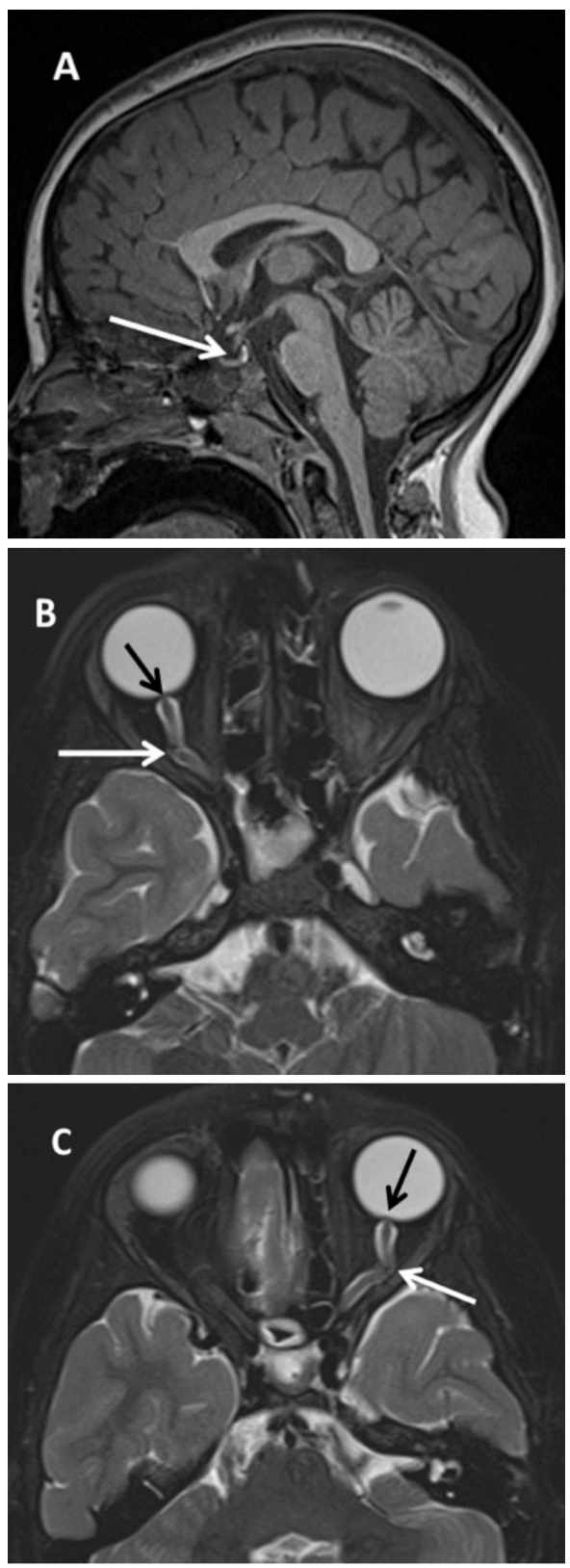

Figure 3 - MRI Brain and orbit for a 10-year-old girl with primary IH. MRI sagittal T1WI brain. A) showing partial empty sella (white arrow). Axial T2 fat saturation for orbits, B\&C) showing flattening of posterior sclera and prominence of the optic nerve head (black arrow) as well as tortuosity of optic nerve and prominent perioptic nerve sheath (white arrow).

with $\mathrm{IH}$, the absence of papilledema does not rule out IH. ${ }^{17,18}$ A previous study reported that papilledema was absent in $48 \%$ of the patients. Interestingly, one of our patients (1.9\%) did not have papilledema at the time of presentation.
Imaging studies are essential in the diagnosis of this condition. A CT scan should be done first, and if the findings are normal, then alumbar puncture with opening pressure should be performed. ${ }^{19}$ Further tests should be considered to rule out secondary causes. Brain MRI is used to rule out intraparenchymal lesions, abnormal meningeal enhancement, or hydrocephalus. ${ }^{20}$ The MRV is used to rule out cerebral venous sinus thrombosis. ${ }^{20}$ MRI findings suggestive of $\mathrm{IH}$ in children include: posterior globe flattening, intraocular protrusion of the optic nerve, horizontal tortuosity of the optic nerve, optic nerve sheath enlargement, and decreased size of the pituitary gland. ${ }^{21,22}$ Lumbar puncture is indicated to measure the CSF opening pressure and to exclude meningitis. ${ }^{1,23}$ Diagnosis of $\mathrm{IH}$ is made according to the modified Dandy criteria. ${ }^{1}$ However, new criteria in children involving a specific CSF opening pressure have been introduced into the literature by Friedman and his colleagues. ${ }^{24}$ The criteria also take into consideration the diagnosis of $\mathrm{IH}$ when the presentation is not clear or atypical. In this study, SIH was more common than PIH, especially in children under 12 years. Moreover, IH was strongly associated with syndromes such as craniosynostosis and Down syndrome or other medical problems such as vitamin D deficiency. Different treatment modalities have been indicated; $; 5,26$ however, the selection of medical, surgical, or combined treatments relies on the severity of the visible signs and symptoms. Medical treatment, including carbonic anhydrase inhibitors, which have been used to lower ICP, is initially indicated. The most commonly used carbonic anhydrase inhibitor is acetazolamide, which is usually used as first-line therapy. ${ }^{1}$ Its use has been thoroughly studied and is thought to decrease production of CSF in the choroid plexus. However, the use of topiramate has not been as thoroughly examined as a monotherapy agent for $\mathrm{IH}$ in adults or children. Topiramate is used for the treatment of $\mathrm{IH}$ through its inhibitory effect on carbonic anhydrase enzyme, which also causes mixed renal tubular acidosis. Moreover, its side effect of appetite suppression makes it an effective adjunct agent for individuals with obesity. ${ }^{27}$ In our study, medical treatment was effective in most cases $(86.8 \%)$, with four cases being treated by topiramate.

Additionally, steroids can be used to reduce intracranial pressure rapidly. Nonetheless, it is only recommended for short-term use in severe cases where surgical intervention is not immediately possible. ${ }^{28}$ In our study, steroids were only used in 4 cases.

Surgical treatment in the form of optic nerve sheath fenestration (ONSF) and CSF diversion is indicated when medical treatment fails to lower ICP, 
or when the visual impairment is deteriorating. ${ }^{16}$ The most commonly used CSF diversion procedures are lumboperitoneal and ventriculoperitoneal shunting, with lumboperitoneal shunting being reported as the most successful in relieving symptoms. ${ }^{16}$ Only three patients in this study required surgical management in the form of lumboperitoneal shunting. ONSF is indicated for acute, severe, or progressive vision loss despite maximum medical treatment. ${ }^{16}$ None of our patients underwent ONSF.

Additionally, lifestyle modifications such as weight reduction in overweight and obese patients can decrease the symptoms associated with IH in adults. However, in the presence of visual deterioration, weight loss alone is insufficient to reduce ICP within the appropriate time. ${ }^{1}$ Interestingly, bariatric surgery can be considered in morbidly obese IH children who failed multiple trials of weight loss, with favorable outcome. ${ }^{29,30}$ During the follow-up period, 7 (13.2\%) cases had a recurrence. Previous studies have reported an $\mathrm{IH}$ recurrence rate in children of up to $18-22 \% .^{31,32}$ Although visual loss in pediatric patients with IH has been previously reported, permanent vision loss in is usually rare. ${ }^{31}$ In our study, most patients did not have any signs or symptoms of visual deterioration.

Study limitation. This study has several limitations, including its retrospective nature and small sample size.

In conclusion, IH in children is rare and differs from that in adults. Unlike in adults, $\mathrm{IH}$ in pre-pubertal children is less commonly associated with obesity, has no female predominance, and more commonly manifests as $\mathrm{SIH}$. On the other hand, $\mathrm{IH}$ in postpubertal children is usually similar to that in adults, and is associated with overweightness as well as female predominance. Our study adds to the body of evidence by reporting on our institutions' experience with IH in the pediatric population.

Acknowledgments. $M$. A. S. was supported by Researchers Supporting Project number (RSP-2019/38), King Saud University, Riyadh, Saudi Arabia.

\section{References}

1. Friedman DI, Jacobson DM. Diagnostic criteria for idiopathic intracranial hypertension. Neurology 2002; 59: 1492-1495.

2. Schexnayder LK, Chapman K. Presentation, investigation and management of idiopathic intracranial hypertension in children. Current Paediatrics 2006; 16: 336-341.

3. Tibussek D, Distelmaier F, Von Kries R, Mayatepek E. Pseudotumor cerebri in childhood and adolescence -- results of a Germany-wide ESPED-survey. Klin Padiatr 2013; 225: 81-85.
4. Bursztyn LL, Sharan S, Walsh L, LaRoche GR, Robitaille J, De Becker I. Has rising pediatric obesity increased the incidence of idiopathic intracranial hypertension in children? Can J Ophthalmol 2014; 49: 87-91.

5. Dessardo NS, Dessardo S, Sasso A, Sarunić AV, Dezulović MS. Pediatric idiopathic intracranial hypertension: clinical and demographic features. Coll Antropol 2010; 34: 217-221.

6. Idiculla T, Zachariah G, Br K, Mohamood N. The incidence and prevalance of idiopathic intracranial hypertension in south Sharaqiah region, Oman. Oman J Ophthalmol 2013; 6: 189-192.

7. Friedman DI, Liu GT, Digre KB. Revised diagnostic criteria for the pseudotumor cerebri syndrome in adults and children. Neurology 2013; 81: 1159-1165.

8. Wall M, Kupersmith MJ, Kieburtz KD, Corbett JJ, Feldon SE, Friedman DI, et al. The idiopathic intracranial hypertension treatment trial: clinical profile at baseline. JAMA Neurol 2014; 71: 693-701.

9. Biousse V, Bruce BB, Newman NJ. Update on the pathophysiology and management of idiopathic intracranial hypertension. J Neurol Neurosurg Psychiatry 2012; 83: 488-494.

10. Rangwala LM, Liu GT. Pediatric idiopathic intracranial hypertension. Surv Ophthalmol 2007; 52: 597-617.

11. Aylward SC, Waslo CS, Au JN, Tanne E. Manifestations of Pediatric Intracranial Hypertension From the Intracranial Hypertension Registry. Pediatr Neurol 2016; 61: 76-82.

12. Spennato P, Ruggiero C, Parlato RS, Buonocore MC, Varone A, Cianciulli E, et al. Pseudotumor cerebri. Childs Nerv Syst 2011; 27: 215-235.

13. Masri A, Jaafar A, Noman R, Gharaibeh A, Ababneh $\mathrm{OH}$. Intracranial Hypertension in Children: Etiologies, Clinical Features, and Outcome. J Child Neurol 2015; 30: 1562-1568.

14. Gradisnik P. Hypoparathyroidism Should Always Be Checked in Papilledema. J Neurosci Rural Pract 2017; 8: 329.

15. Moavero R, Sforza G, Papetti L, Battan B, Tarantino S, Vigevano F, et al. Clinical Features of Pediatric Idiopathic Intracranial Hypertension and Applicability of New ICHD-3 Criteria. Front Neurol 2018; 9: 819.

16. Ko MW, Liu GT. Pediatric idiopathic intracranial hypertension (pseudotumor cerebri). Horm Res Paediatr 2010; 74: 381-389.

17. Faz G, Butler IJ, Koenig MK. Incidence of papilledema and obesity in children diagnosed with idiopathic "benign" intracranial hypertension: case series and review. J Child Neurol 2010; 25: 1389-1392.

18. Aylward SC, Aronowitz C, Roach ES. Intracranial Hypertension Without Papilledema in Children. J Child Neurol 2016; 31 : 177-183.

19. Rogers DL. A review of pediatric idiopathic intracranial hypertension. Pediatr Clin North Am 2014; 61: 579-590.

20. Bidot S, Saindane AM, Peragallo JH, Bruce BB, Newman NJ, Biousse V. Brain Imaging in Idiopathic Intracranial Hypertension. J Neuroophthalmol 2015; 35: 400-411.

21. Gorkem SB, Doganay S, Canpolat M, Koc G, Dogan MS, Per $\mathrm{H}$, et al. MR imaging findings in children with pseudotumor cerebri and comparison with healthy controls. Childs Nerv Syst 2015; 31: 373-380.

22. Kwee RM, Kwee TC. Systematic review and meta-analysis of MRI signs for diagnosis of idiopathic intracranial hypertension. Eur J Radiol 2019; 116: 106-115.

23. Cleves-Bayon C. Idiopathic Intracranial Hypertension in Children and Adolescents: An Update. Headache 2018; 58: 485-493. 
24. Friedman DI, Liu GT, Digre KB. Revised diagnostic criteria for the pseudotumor cerebri syndrome in adults and children. Neurology 2013; 81: 1159-1165.

25. Albakr A, Hamad MH, Alwadei AH, Bashiri FA, Hassan HH, Idris $\mathrm{H}$, et al. Idiopathic intracranial hypertension in children: Diagnostic and management approach. Sudan J Paediatr 2016; 16: 67-76.

26. Mollan SP, Davies B, Silver NC, Shaw S, Mallucci CL, Wakerley $\mathrm{BR}$, et al. Idiopathic intracranial hypertension: consensus guidelines on management. J Neurol Neurosurg Psychiatry 2018; 89: 1088-1100.

27. Celebisoy N, Gokcay F, Sirin H, Akyurekli O. Treatment of idiopathic intracranial hypertension: topiramate vs acetazolamide, an open-label study. Acta Neurol Scand 2007; 116: 322-327.
28. Standridge SM. Idiopathic intracranial hypertension in children: a review and algorithm. Pediatr Neurol 2010; 43: 377-390.

29. Pratt JS, Browne A, Browne NT, Bruzoni M, Cohen M, Desai A, et al. ASMBS pediatric metabolic and bariatric surgery guidelines, 2018. Surg Obes Relat Dis 2018; 14: 882-901.

30. Handley JD, Baruah BP, Williams DM, Horner M, Barry J, Stephens JW. Bariatric surgery as a treatment for idiopathic intracranial hypertension: a systematic review. Surg Obes Relat Dis 2015; 11: 1396-1403.

31. Ravid S, Shahar E, Schif A, Yehudian S. Visual Outcome and Recurrence Rate in Children With Idiopathic Intracranial Hypertension. J Child Neurol 2015; 30: 1448-1452.

32. Aylward SC, Way AL. Pediatric Intracranial Hypertension: a Current Literature Review. Curr Pain Headache Rep 2018; 22: 14.

\section{Authorship entitlement}

Excerpts from the Uniform Requirements for Manuscripts Submitted to Biomedical Journals updated November 2003.

Available from www.icmje.org

The international Committee of Medical Journal Editors has recommended the following criteria for authorship; these criteria are still appropriate for those journals that distinguish authors from other contributors.

Authorship credit should be based on 1) substantial contributions to conception and design, or acquisition of data, or analysis and interpretation of data; 2) intellectual content; and 3) final approval of the version to be published. Authors should meet conditions 1,2 , and 3 .

Acquisition of funding, collection of data, or general supervision of the research group, alone, does not justify authorship.

An author should be prepared to explain the order in which authors are listed. 